\title{
CONVERSÃO DE ÁRVORES EM MULTIPRODUTOS DA MADEIRA UTILIZANDO PROGRAMAÇÃO INTEIRA ${ }^{1}$
}

\author{
Bráulio Pizziôlo Furtado Campos², Daniel Henrique Breda Binoti ${ }^{3}$, Márcio Lopes da Silva ${ }^{4}$, Hélio Garcia
} Leite $^{4}$ e Mayra Luiza Marques da Silva Binoti ${ }^{5}$

\begin{abstract}
RESUMO - Este estudo foi conduzido visando aumentar a renda e a segurança de um empreendimento florestal através da conversão de árvores em multiprodutos de madeira utilizando programação inteira. Os dados utilizados são referentes a um plantio de um clone híbrido de Eucalyptus grandis x Eucalyptus urophylla, aos 7 anos de um produtor rural na zona da mata em Minas Gerais. Para realização da conversão otimizada, foram utilizadas informações de alternativas de comercialiação da madeira produzida no povoamento e das características dos produtos. Foram consideradas 4 alternativas de destino das toras: energia, celulose, escora e serraria, com diferentes dimensões requeridas quanto aos diâmetros mínimos e máximos e o comprimento das toras. O problema se resumiu em atender a combinação otimizada baseado em dois cenários propostos: a) Maximização do volume total obtido, e b) otimização da receita global obtida. A fim comparativo, destinou-se a floresta a um único produto sem estudo de otimização. A comparação dos resultados obtidos demonstra os benefícios econômicos obtidos pela conversão do plantio em multiprodutos quando comparado a uso único, tendo um acréscimo médio de 33,69\% na receita média obtida por apenas um produto. A maximização da receita total obtida teve um acréscimo de 20,6\% e 16,3\% quando comparada a receita obtida pela destinação do povoamento para escora e pela maximização do volume respectivamente. Logo, conclui-se que a otimização da receita global demonstrouse ser a melhor alternativa de remuneração advinda do povoamento florestal.
\end{abstract}

Palavras-Chave: Multiprodutos; Programação inteira; Sortimento florestal.

\section{CONVERSION OF WOOD TREES IN MULTIPLE PRODUCTS USING INTEGER PROGRAMMING}

\begin{abstract}
The objective of this study was to increase the income and safeness of a forestry enterprise by converting trees into wood multiproduct using integer programming. The data used in this experiment are related to the planting of a hybrid clone of Eucalyptus grandis $x$ Eucalyptus urophylla, at 7 years of age of a farmer from Minas Gerais, Brazil. To perform the optimized conversion, information from alternative commercialization of the wood produced in the settlement and the characteristics of products were used. Four alternative destinations of the logs were taken into account: energy, cellulose, anchor and sawmill, with different dimensions and the required minimum and maximum diameters and length of logs. The problem consisted in meeting the optimal combination based on two proposed scenarios: a) maximization of the total volume obtained, and b) optimization of the overall revenue obtained. The comparative order was destined to a single forest product with no study of optimization. The comparison of results demonstrates the economic benefits achieved by planting in multiproduct conversion when compared to single use, with an average increase
\end{abstract}

\footnotetext{
${ }^{1}$ Recebido em 12.04.2012 aceito para publicação em 13.08.2013.

${ }^{2}$ Programa de Pós-Graduação em Ciências Florestais na Universidade Federal do Espírito Santo, UFES, Brasil. E-mail: <brauliopfc@hotmail.com>.

${ }^{3}$ Programa de Pós-Graduação em Ciência Florestal na Universidade Federal de Viçosa, UFV, Brasil. E-mail: <danielhbbinoti@gmail.com>.

${ }^{4}$ Departamento de Engenharia Florestal, Universidade Federal de Viçosa, UFV, Brasil. E-mail: <marlosil@ufv.br>e <hgleite@gmail.com>.

${ }^{5}$ Departamento de Engenharia Florestal, Universidade Federal dos Vales do Jequitinhonha e Mucuri, UFVJM, Brasil. E-mail: <mayrabinoti@gmail.com>.
} 
of $33.69 \%$ in average revenue achieved by just one product. The maximization of total revenue obtained had an increase of $20.6 \%$ and $16.3 \%$ compared to revenues obtained by the allocation of settlement anchor and maximization of the volume respectively. Therefore, it is concluded that optimization of the overall revenue proved to be the best choice for income from forest settlement.

Keywords: Assortment forest; Integer programming; Multiproduct

\section{INTRODUÇÃO}

O planejamento de atividades é fundamental para qualquer empresa que está num ambiente competitivo, pois possibilita o estabelecimento de prioridades e a definição de estratégias atuais para obtenção de resultados satisfatórios no futuro. Este fato torna-se extremamente marcante na atividade florestal que apresenta longos ciclos de produção.

O mercado de produtos sólidos é, em geral, abastecido por madeiras nobres, muitas das quais protegidas por lei, e exploradas de forma irregular em florestas nativas. Para diminuição dessa pressão predatória, práticas como desbastes e desrama passaram a ser estudadas e aplicadas, sobretudo em povoamentos de Pinus, nos quais foram realizados maiores estudos sobre definição e aplicação de desbastes destacando-se os estudos de Silva (1970); Soares (1970); Campos (1980); Bertoloti et al. (1983). Estudos sobre desbastes em povoamentos de eucalipto são mais recentes, destacando-se os trabalhos de Nogueiraet al. (2001), Nogueira (2003), Dias (2005), Leite et al., (2010) e Binoti et al. (2010). Com a demanda crescente por produtos madeireiros, aliada à redução ou estagnação da área plantada com pinus, a utilização de eucalipto para produção de madeira de grandes dimensões, para fins estruturais e uso na serraria, é uma realidade presente.

A fim de se estimar a produção futura de povoamentos florestais, o planejador florestal possui diversas ferramentas que vão desde simples inventários florestais contínuos e modelos de crescimento e produção a modernas ferramentas de Inteligência Artificial (CAMPOS; LEITE, 2009).

A utilização de inventários florestais contínuos (IFC) é rotina na maioria das empresas florestais, e com as informações oriundas destes, os povoamentos são avaliados aplicando-se os planos de manejo em curto prazo. Com as informações obtidas pelos IFC, consegue-se determinar a qualidade e a quantidade dos produtos madeireiros a serem obtidos.
A definição dos produtos a serem obtidos de povoamentos de florestais é uma atividade relativamente complexa, sendo influenciado por diversos fatores, dentre os quais destacam-se: características e especificações cada produto (comprimento e diâmetros mínimos e máximos), receita obtida, custo de processamento, características operacionais, demanda de mercado, dentre outras. Nesse cenário, faz-se necessário a utilização de técnicas de otimização para a definição das estratégias a serem adotadas em cada povoamento para a obtenção do máximo de rendimento econômico para o empresário.

Devido à enorme complexidade e quantidade de variáveis envolvidas nesses modelos, diversas ferramentas da pesquisa operacional foram introduzidas na área florestal, possibilitando a redução de riscos e incertezas na tomada de decisão (RODRIGUES, 2001). As técnicas de otimização mais comumente usadas para a definição das estratégias ótimas de corte de árvores são Programação Dinâmica (PD), Programação em Redes e simulação sendo que técnicas com Programação Inteira e Programação Linear são menos utilizadas (MARSHALL, 2005).

A utilização da Programação Dinâmica para a conversão de toras em multiprodutos apresenta destaque dentre as outras técnicas de pesquisa operacional, podendo ser citados os trabalhos de Leite et al. (1995a), Lima et al. (1997), Chichorro (2000), Soares et al. (2003), e Soares et al. (2004). Contudo a utilização de técnicas de PD não garante a obtenção do padrão de corte ótimo para todo o povoamento. Desta forma técnicas de Programação Inteira Mista (PIM) e heurísticas podem ser utilizadas para o desenvolvimento e geração de estratégias ótimas de corte para todo o povoamento, permitindo ainda a imposição de restrições para determinados produtos.

Em função das poucas aplicações da PIM na obtenção de multiprodutos de plantios florestais, objetivou-se nesse trabalho desenvolver e demonstrar a aplicação de técnicas de PIM para a obtenção de múltiplos produtos de um plantio florestal. 


\section{MATERIAL E MÉTODOS}

\subsection{Dados}

Os dados utilizados nesse estudo são característicos de um pequeno produtor florestal, localizado na zona da mata de Minas Gerais. O produtor possui um plantio de 300 ha de um clone híbrido de Eucalyptus grandis x Eucalyptus urophylla, com idade média de 7 anos. O produtor em questão possui diversas possibilidades de comercialização para os produtos obtidos de seu plantio.

A implantação do plantio florestal foi realizada por meio de programa de fomento florestal, sendo que, como exigência contratual o produtor deve repassar no mínimo $30 \mathrm{~m}^{3} / \mathrm{h}$ a ao programa de fomento. Para a análise da conversão do plantio em questão em multiprodutos gerou-se os cenários a) Maximização do volume total obtido, e b) otimização da receita global obtida. Como comparativo avaliou-se a conversão do povoamento em um único produto, para celulose, energia e escora, sendo estes cenários não otimizados. As principais informações do povoamento em questão são apresentadas abaixo.

\subsection{Distribuição de Diâmetros}

A distribuição diamétrica do povoamento em questão bem como a altura estimada para cada classe é apresentada na Tabela 1.

\subsection{Modelo de taper}

Utilizou-se o modelo de Kozak descrito abaixo (CAMPOS; LEITE, 2009).

$$
d_{i=} \operatorname{dap} \sqrt{\beta_{0}+\beta_{1}\left(\frac{h_{i}}{H t}\right)+\beta_{2}\left(\frac{h_{i}}{H t}\right)^{2}}+\varepsilon_{i}
$$

em que, $d_{i}=$ Diâmetro comercial na altura $\mathrm{h}_{\mathrm{i}}(\mathrm{cm})$; dap = Diâmetro medido a 1,30 m de altura do solo $(\mathrm{cm})$; $h_{i}=$ Altura onde ocorre o diâmetro comercial d $(\mathrm{m})$; $H t=$ Altura total (m); $\beta_{i}$ os parâmetros a serem estimados; e $\varepsilon_{i}$ o erro aleatório.
Logo, a equação após o ajuste se encontra da seguinte forma:

$$
d_{i=} \operatorname{dap} \sqrt{1,22808-2,14658\left(\frac{h_{i}}{H t}\right)+0,98868\left(\frac{h_{i}}{H t}\right)^{2}}
$$

\subsection{Produtos}

Os produtos que podem comercializados pelo produtor são apresentados na Tabela 2 .

\subsection{Programação Inteira Mista}

A formulação do problema será baseada na geração de alternativas de toragem para cada classe de diâmetro, sendo esta em função da forma da árvore e das dimensões dos sortimentos e produtos. Na formulação do modelo de PIM, a variável de decisão $X_{i j}$ representa o j-ésimo padrão de corte atribuído a i-ésima classe de diâmetro. Para cada classe de diâmetro são geradas todas as possíveis alternativas de toragem que possam ser adotadas.

Os modelos utilizados nesse trabalho foram gerados pelo software OtimToras (desenvolvidos por Leite, H.G.; Binoti, D.H.B.; Binoti, M.L.M.S). Os modelos gerados foram resolvidos pelo algoritmo Branch-and-Bound com a versão demonstrativa do software lingo.

\subsection{Função objetivo}

A função objetivo para o cenário A, tendo como objetivo a maximização do volume útil total pode ser descrita como:

$$
\operatorname{Max} . Z=\sum_{i=1}^{m} \sum_{j=1}^{n} c_{i j} x_{i j}
$$

em que, $\mathrm{Z}=$ Volume Total $\left(\mathrm{m}^{3}\right), x_{i j}=$ Variáveis de Decisão, $j$-ésima alternativa de corte adotada na $i$-ésima classe de diâmetro; $c_{i j}=$ volume útil por cada classe de diâmetro $i$, seguindo a alternativa de corte $j ; m=$ Número total de classe de diâmetro; $n$ = é número total de alternativas de corte para a $i$-ésima classe de diâmetro.

Tabela 1 - Distribuição de diâmetros observada e altura estimada para os dados do povoamento estudado por hectare. Table 1 -Distribution of diameters observed and estimated height for the data on the population studied per ha.

\begin{tabular}{lcccccccccc}
\hline & \multicolumn{1}{c}{ Classe de DAP } \\
\cline { 2 - 9 } PROJETO & 5 & 7 & 9 & 11 & 13 & 15 & 17 & 19 \\
\hline Número & 7 & 15 & 33 & 97 & 280 & 481 & 148 & 26 \\
Altura & 11,4 & 15,1 & 17,7 & 19,5 & 20,9 & 22,0 & 22,9 & 23,6 & 24,2 \\
\hline
\end{tabular}


Tabela 2 - Características dos produtos comercializáveis pelo produtor. Table 2 - Characteristics of marketable products by the producer.

\begin{tabular}{lccccc}
\hline Produtos & Comprimento $(\mathrm{cm})$ & Diâmetro Mínimo & Diâmetro Máximo & Rendimento & R\$/m ${ }^{3}$ \\
\hline Energia & 220 & 4 & 40 & 0,98 \\
Celulose & 600 & 8 & 30 & 0,95 \\
Escora & 350 & 8 & 25 & 0,98 \\
Serraria & 300 & 15 & 50 & 0,95 \\
\hline
\end{tabular}

A função objetivo para o cenário B, tendo como objetivo a maximização do valor presente líquido total pode ser descrita como:

$$
\operatorname{Max} . Z=\sum_{i=1}^{m} \sum_{j=1}^{n} c_{i j} x_{i j}
$$

em que, $\mathrm{Z}=$ Valor Presente Líquido Total $(\mathrm{R} \$), \mathrm{c}_{i j}=$ valor presente líquidopor cada classe de diâmetro $i$, seguindo a alternativa de corte $j$.

\subsection{Restrição de classe de diâmetro}

Como as prescrições geradas após a otimização serão de acordo com a classe de diâmetro, esta restrição impede que uma árvore tenha um número de alternativas de corte maior que as alternativas de sua classe diamétrica. Logo a expressão de restrição de diâmetro será:

$$
\begin{gathered}
\sum_{i=1}^{n} x_{i j}=1 \forall i \\
x_{i j} \in\{0,1\}
\end{gathered}
$$

Logo se assumir valor 1 a $j$-ésima será adotada a alternativa na $i$-ésima classe de diâmetro.

\subsection{Restrições de demanda}

Tendo em vista quantidades requeridas de cada produto pelo mercado, impõe-se restrições de demanda onde cada produto terá um máximo e um mínimo de volume requerido em um período de tempo, sendo gerada pelas seguintes expressões:

$$
\begin{aligned}
& \sum_{i=1}^{m} \sum_{j=1}^{n} v_{i j k} x_{i j} \geq D \min _{k}\{k=0,1, \ldots, H-1\} \\
& \sum_{i=1}^{m} \sum_{j=1}^{n} v_{i j k} x_{i j} \leq D \max _{k}\{k=0,1, \ldots, H-1\}
\end{aligned}
$$

em que, $x_{i j}=$ Variáveis de decisão, representando a $j$-ésima alternativa de corte adotada na $i$-ésima classe de diâmetro; $m$ = Número total classes de diâmetro; $n=$ Número total de alternativas de corte para a $i$-ésima classe de diâmetro; $V_{i j k}=$ Volume $\left(\mathrm{m}^{3}\right)$ produzido por classe de diâmetro, do produto $k$, pela $i$-ésima classe de diâmetro adotando-se a $j$-ésima alternativa de corte; $\operatorname{Dmin}_{k}$ e $\operatorname{Dmax}_{k}=$ Demandas volumétricas $\left(\mathrm{m}^{3}\right)$ mínima e máxima respectivamente, de cada produto. Para o problema em questão deseja-se somente limitar o volume para celulose acima de $30 \mathrm{~m}^{3} /$ ha como exigência do programa de fomento adotado.

\section{RESULTADOS}

Foram gerados 3623 padrões de corte distintos, para ambos os cenários avaliados. Na Tabela 3 são apresentados os principais resultados dos modelos obtidos para os cenários avaliados.

O comparativo dos volumes totais obtidos pela maximização do volume total, bem como o comparativo da receita total obtidapela maximização da receita total é apresentado nas Figuras 1 e 2, respectivamente.

\section{DISCUSSÃO}

Esse trabalho objetivou desenvolver e demonstrar a aplicação de técnicas de programação inteira mista para a obtenção de múltiplos produtos de um plantio florestal. Como objetivo, comparou-sea maximização da receita total obtida e o volume total obtido, tendo como comparativo a conversão do povoamento em um único produto.

A comparação dos resultados obtidos demonstra os benefícios econômicos obtidos pela conversão do plantio em multiprodutos. A conversão em multiprodutos teve um acréscimo médio de 65,08\% na receita quando comparado à receita média obtida pela conversão do povoamento em um único produto. A maximização da 
Tabela 3 - Resumo dos resultados obtidos pelos cenários simulados ( $\mathrm{m}^{3} / \mathrm{ha}$ ). Table 3 - Summary of results obtained by the simulated scenarios $\left(\mathrm{m}^{3} / \mathrm{ha}\right)$.

\begin{tabular}{|c|c|c|c|c|c|c|c|c|}
\hline \multirow[t]{2}{*}{ Cenário } & \multirow{2}{*}{$\begin{array}{c}\text { Classe de } \\
\text { Diâmetro }(\mathrm{cm})\end{array}$} & \multicolumn{4}{|c|}{ Produtos } & \multirow{2}{*}{$\begin{array}{l}\text { Receita } \\
\text { (R\$/ha) }\end{array}$} & \multirow{2}{*}{$\begin{array}{c}\text { Volume } \\
\text { Total (m³/ha) }\end{array}$} & \multirow{2}{*}{$\begin{array}{l}\text { Resíduo } \\
\text { ( } \mathrm{m}^{3} / \mathrm{ha} \text { ) }\end{array}$} \\
\hline & & Energia & Celulose & Escora & Serraria & & & \\
\hline \multirow[t]{10}{*}{ A } & 5,00 & 0,03 & 0,00 & 0,00 & 0,00 & 1,49 & 0,03 & 0,03 \\
\hline & 7,00 & 0,30 & 0,00 & 0,00 & 0,00 & 14,99 & 0,30 & 0,07 \\
\hline & 9,00 & 0,86 & 0,00 & 0,73 & 0,00 & 101,43 & 1,59 & 0,07 \\
\hline & 11,00 & 2,54 & 0,00 & 5,52 & 0,00 & 568,44 & 8,06 & 0,14 \\
\hline & 13,00 & 7,90 & 14,02 & 13,30 & 0,00 & 2370,20 & 35,22 & 0,31 \\
\hline & 15,00 & 32,48 & 0,00 & 54,59 & 0,00 & 5991,17 & 87,07 & 0,06 \\
\hline & 17,00 & 15,22 & 8,36 & 12,19 & 0,00 & 2280,39 & 35,78 & 0,00 \\
\hline & 19,00 & 1,54 & 6,42 & 0,00 & 0,00 & 494,44 & 7,96 & 0,01 \\
\hline & 21,00 & 0,06 & 1,22 & 0,25 & 0,00 & 102,51 & 1,53 & 0,00 \\
\hline & Total & 60,93 & 30,03 & 86,58 & 0,00 & 11925,07 & 177,54 & 0,70 \\
\hline \multirow[t]{10}{*}{$\mathrm{B}$} & 5,00 & 0,03 & 0,00 & 0,00 & 0,00 & 1,49 & 0,03 & 0,03 \\
\hline & 7,00 & 0,30 & 0,00 & 0,00 & 0,00 & 14,99 & 0,30 & 0,07 \\
\hline & 9,00 & 0,86 & 0,00 & 0,73 & 0,00 & 101,43 & 1,59 & 0,07 \\
\hline & 11,00 & 2,96 & 4,86 & 0,00 & 0,00 & 464,17 & 7,82 & 0,22 \\
\hline & 13,00 & 5,86 & 0,00 & 29,10 & 0,00 & 2621,22 & 34,96 & 0,57 \\
\hline & 15,00 & 10,08 & 22,47 & 52,92 & 0,00 & 6197,95 & 85,47 & 0,60 \\
\hline & 17,00 & 4,72 & 0,00 & 20,46 & 10,32 & 3421,02 & 35,50 & 0,03 \\
\hline & 19,00 & 0,71 & 2,34 & 0,78 & 4,05 & 857,50 & 7,88 & 0,03 \\
\hline & 21,00 & 0,07 & 0,33 & 0,10 & 1,02 & 186,02 & 1,52 & 0,00 \\
\hline & Total & 25,58 & 30,00 & 104,09 & 15,39 & 13865,80 & 175,07 & 1,65 \\
\hline
\end{tabular}

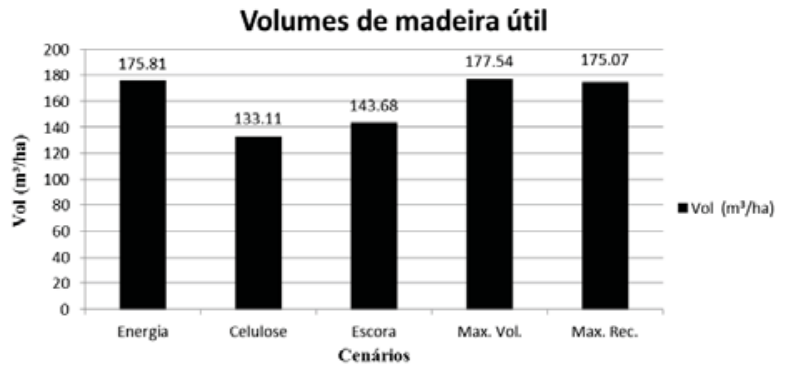

Figura 1 - Volume total obtido por hectare dos cenários simulados.

Figure 1 - Total volume obtained per hectare from simulated scenarios.

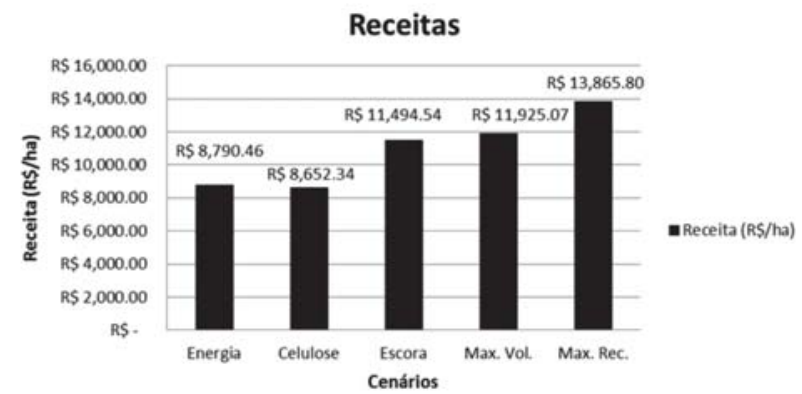

Figura 2-Receita total obtida pelos cenários simulados (R $\$ /$ ha). Figure 2 - Total revenue obtained by the simulated scenarios $(R \$ / h a)$. receita total obtida teve um acréscimo $20,6 \%$ e 16,3\% quando comparada a receita obtida pela destinação do povoamento escora e pela maximização do volume respectivamente.

Soares et al. (2003) empregaram a programação dinâmica para otimização de multiprodutos de madeira de povoamentos de eucalipto, considerando diferentes sortimentos. O uso da PD permitiu a obtenção de maior retorno econômico da madeira em usos distintos. Contudo, a aplicação da PD em nível de árvore pode não garantir a obtenção de soluções ótimas considerando todas as características do empreendimento florestal.

O trabalho em questão demonstra uma ferramenta matemática de maximização dos retornos econômicos que podem ser obtidos por pequenos proprietários florestais. Os modelos matemáticos foram desenvolvidos utilizando o sistema OtimToras, que possui como principal característica ser distribuído gratuitamente pelos autores. Dessa forma as técnicas matemáticas demonstradas são apresentadas de forma amigável ao usuário final, possibilitando a utilização do sistema por extensionista e pequenos produtores.

Revista Árvore, Viçosa-MG, v.37, n.5, p.881-887, 2013 


\section{CONCLUSÃO}

A Programação Inteira Mista demonstrou-se uma ferramenta de grande utilidade para conversão de árvores em multiprodutos, principalmente quando se observa alguma restrição de demanda dos produtos a serem obtidos. Houve um acréscimo médio de 33,69\% da receita e 16,86\% do volume útil quando se propôs a conversão do povoamento em multiprodutos, aumentando a viabilidade e a concorrência das plantações florestais com outras culturas.

Os cenários de Maximização da Receita demonstrou-se a melhor alternativa de remuneração advinda do povoamento florestal.

Mesmo com os estudos de maximização, deve-se fazer uma análise extra dos resultados, observando questões de logística, transporte e a compatibilidade do tipo de madeira com o produto destinado, os quais podem alterar os valores encontrados.

\section{AGRADECIMENTO}

Ao CNPq pela concessão de bolsas, à FAPEMIG pelo apoio financeiro e à UFV pela infra-estrutura e pessoal disponibilizados para realização deste presente trabalho.

\section{REFERÊNCIAS}

BERTOLOTTI, G. et al. Efeitos de diferentes métodos e intensidades de desbaste na produtividade de Pinus caribaea var. hondurensis Barr. et Golf. IPEF, Piracicaba, v.24, p.47-54, 1983.

BINOTI, D. H. B. Estratégias de regulação de florestas equiâneas com vistas ao manejo da paisagem. 2010. 159f.Dissertação (Mestrado em Ciência Florestal) -Universidade Federal de Viçosa, Viçosa, MG, 2010.

CAMPOS, J. C. C. Análise de crescimento e produção em plantações desbastadas. Revista Árvore, v.4, n.2, p.157-169, 1980.

CAMPOS, J. C. C.; LEITE, H. G. Mensuração florestal: perguntas e respostas. 3.ed. Viçosa, MG, Universidade Federal de Viçosa, 2009. 548p.

CHICHORRO, J. F.Análise estrutural e econômica de multiprodutos da madeira. 2000. 241f. Tese (Doutorado em Ciência Florestal) - Universidade Federal de Viçosa, Viçosa, MG, 2000.
DIAS, A. N. Um modelo para gerenciamento de plantações de eucalipto submetidas a desbaste. 2005. 135f. Tese (Doutorado em Ciência Florestal) Universidade Federal de Viçosa, Viçosa, MG, 2005.

LEITE, H. G.; CAMPOS, J. C. C.; PAULA JUNIOR, G. G. Emprego de um modelo de programação dinâmica para conversão de troncos em multiprodutos da madeira. Revista Árvore, v.19, n.4, p.447-465, 1995a.

LEITE, H. G.et al. Avaliação do ajuste das funções Weibull e hiperbólica a dados de povoamentos de eucalipto submetidos a desbaste. Revista Árvore, v.34, n.2, p.305-311, 2010.

LIMA, D. G.et al. Um modelo de suporte a decisão sobre multiprodutos de povoamentos de eucalipto. Revista Árvore, v.21, n.1, p.35-48, 1997.

MARSHALL, H. D. An investigation of factors affecting the optimal output log distribution from mechanical harvesting and processing systems. 2005. 210f.(PhD thesis) - Oregon StateUniversity, Portland, 2005.

NOGUEIRA, G. S.et al.Determinação da idade técnica de desbaste em plantações de eucalipto utilizando o método dos ingressos percentuais. Scientia Forestalis, n.59, p.51-59, 2001.

NOGUEIRA, G. S. Modelagem do crecimento e da produção de povoamentos de Eucalyptus spp e de Tectona grandis submetidas a desbaste. 2003. 126f. Tese (Doutorado em Ciência Florestal) Universidade Federal de Viçosa, Viçosa, MG,2003.

RODRIGUES, F. L. Metaheurística e sistema de suporte à decisão no gerenciamento de recursos florestais. 2001. 225f. Tese (Doutorado em Ciência Florestal) - Universidade Federal de Viçosa, Viçosa, MG, 2001.

SILVA, J. C. Aspectos do desbaste em reflorestamento no norte do Paraná. Floresta, v.2, n.3, p.13-8, 1970.

SOARES, R. V. Desbastes em araucária angustifólia (Bert) O’Ktze. Floresta, v.2, n.3, p.59-67, 1970. 
SOARES, T. S.et al. Avaliação econômica de plantações de Eucalipto submetidas a

desbaste. Revista Árvore, v.27, n.4, p.481486, 2003.
SOARES, T. S.et al.Uso de diferentes alternativas para viabilizar a relação hipsométrica no povoamento florestal. Revista Árvore, v.28, n.6, p.845-854, 2004. 
